\title{
Extended Cooperative Balanced Space-Time Block Coding for Increased Efficiency in Wireless Sensor Networks (Work in Progress)
}

\author{
Ali Ekşim ${ }^{1}$ and Mehmet Ertuğrul Çelebi ${ }^{2}$ \\ ${ }^{1}$ Tubitak-UEKAE, P.K. 74, 41470, Gebze, Kocaeli, Turkey \\ alieksim@uekae.tubitak.gov.tr \\ ${ }^{2}$ Department of Electronics and Communication Engineering, \\ Istanbul Technical University, Maslak, Istanbul, Turkey, 34469 \\ celebi@ehb.itu.edu.tr
}

\begin{abstract}
Diversity techniques for communications among sensors are very effective tool to increase reception quality and battery lifetimes. A well-known method to increase diversity in cooperative communications is sensor (relay) selection. However, sensor selection method may lead to the selection of the same (near) sensor for transmission over a long period. One of the alternative techniques to sensor selection is cooperative balanced space-time block coding which utilizes every sensor in sight, thus, distributes the energy consumption among many sensors. Furthermore, it guarantees full diversity for any number of relay sensors. In this work, we consider dual-hop amplify-and-forward wireless sensor network and extend the cooperative balanced space-time block code family to improve its performance. In the proposed scheme, a larger number of codes can be generated for improved coding gain, and better signal-to-noise ratio improvement can be obtained compared to sensor selection schemes.
\end{abstract}

Keywords: Balanced space-time block codes, Cooperative communications, Relay selection, Wireless sensor networks.

\section{Introduction}

Recently, the desire for connectivity has caused an exponential growth in wireless sensor networks. These networks support a wide variety services while maintaining low power consumption and increased spectrum efficiency. On the other hand, they suffer from fading which causes severe variation in signal attenuation. These constraints can be met via diversity techniques which combine independent fading copies of transmitted signal at the receiver [1]. One of the well-known diversity methods in MIMO systems is transmit antenna diversity which does not especially increase transmission bandwidth and power. Space-time coding methods have been developed to utilize the transmit diversity potential [2-5].

* The work of Ali Ekşim is supported partially by the European Comission (EC), FP-7 Project ICE, Project No. 206546. The work of Mehmet Ertuğrul Çelebi is supported partially by the Scientific and Technological Research Council of Turkey (TUBITAK), Project No.107E022. 
One of the space-time coding scheme is Orthogonal Space-Time Block Codes (OSTBCs) which provide full diversity advantages with a low decoding complexity. The transmitted symbols are decoded separately using linear processing [5]. However, full diversity and full rate for more than two antennas cannot be achieved with OSTBCs. Several quasi-orthogonal STBCs that provide full rate at the expense of some loss in diversity [6],[7] and OSTBCs that provide full diversity with some loss in code rate [5], [8] have been proposed in the literature. In [4], full rate Balanced Space-Time Block Codes (BSTBCs) have been proposed which achieve full diversity for arbitrary number of transmit antennas when one or more feedback bits are transmitted via feedback channel.

Due to insufficient antenna space, cost and hardware limitations, wireless sensors may not be able to support multiple transmit antennas. To overcome this difficulty, recently, with the increasing interests in sensor networks, researchers have been looking for methods to exploit spatial diversity using the antennas of different units in the network. This type of diversity is called cooperative diversity [9-13] where virtual antenna arrays can be formed to overcome the drawback of channel correlation and space limitations of mobile units. Cooperative diversity reduces the required transmit power which leads to longer battery life, and increases capacity in interference limited systems.

In [14], Cooperative Balanced Space-Time Block Codes (CBSTBCs) have been proposed where the number of relay sensors can be arbitrary. CBSTBCs cause uniform energy consumption over relay sensors and increase the wireless sensor network lifetime. However, in the proposed scheme, the number of CBSTBCs is limited. For example, if three or four sensors are active in the environment, the number of available codes is only six and twenty-eight, respectively. It can be seen that extension of available coding family increase the efficiency.

In this paper, we propose the Extended Cooperative Balanced Space-Time Block Codes (ECBSTBCs) scheme. With the help of the feedback information, arbitrary number of ECBSTBCs can be utilized. The reason for this extension is to improve upon relay (sensor) selection methods [17] in terms of bit-error performance and energy consumption. In this regard, in the second section, the system model is described, in the third section, the ECBSTBCs are explained, in the fourth section, performance analysis is presented, and in the last section, the results of the paper and the conclusion are given.

The following notation used in the paper: * denotes the conjugate operation; $\operatorname{Re}\{$. and $\operatorname{Im}\{$.$\} are the real and imaginary part of the argument, respectively. \operatorname{diag}\left\{d_{l}, . ., d_{N}\right\}$ is the $N \mathrm{x} N$ diagonal matrix whose $i$ th diagonal entry is $d_{i} . P\left(s_{k} \rightarrow \hat{s}_{k}\right)$ is the Pairwise Error Probability (PEP) of deciding in favor of symbol $\hat{s}_{k}$ when $s_{k}$ is transmitted. The operator $E($.$) and \exp ($.$) indicate the statistical expectation and the exponential of the$ elements of the argument, respectively. The operator ceil\{.\} rounds to the largest integer less or equal than its argument.

\section{System Model}

The wireless sensor network consists of one source sensor, one fusion center and $N$ relay sensors which are located randomly and independently. All sensors are equipped 
with a single antenna. They cannot transmit and receive at the same time. The relay sensors utilize amplify and forward cooperative protocol [9]. All channels are assumed frequency flat Rayleigh fading channel where channel gains are circularly complex Gaussian random variables and statistically independent from each other. The channels are quasi-static, namely, the fading coefficients remain constant over the duration of one frame. $h_{s r i}$ is the channel coefficient from the source sensor to the $i$ th relay sensor and $h_{\text {rid }}$ is the channel coefficient from the $i$ th relay sensor to the fusion center where $i=1,2, . ., N$.

The fusion center is assumed to have perfect knowledge of the relay-fusion center channels. It is also assumed that the fusion center has no knowledge of the source sensor-relay sensor channels, since perfect knowledge of the source sensor-relay sensor channels at the fusion center requires considerable protocol overhead. Each sensor is assumed to have perfect knowledge of its own source sensor-relay sensor channel. $T$ is the coherence interval, $P_{1}$ is the average transmit power of the source sensor, and $P_{2}$ is the average total transmit power of the relay sensors. The source sensor data bits are mapped by streams of $y$ bits into $M$-PSK symbols where $M=2^{y}$.

\section{Extended Cooperative Balanced Space-Time Block Codes}

The ECBSTBCs can be obtained with an OSTBC multiplied by an extension matrix. Since Alamouti's code is the only orthogonal code with rate one and minimum delay, the ECBSTBCs can be obtained as an extension of the Alamouti`s code [15]

$$
C=X W \text {. }
$$

Here $\boldsymbol{X}$ is the Alamouti's code matrix, $\boldsymbol{W}$ is a $2 \mathrm{x} N(N>2)$ matrix whose columns are $2 \times 1$ standard basis vectors, and the rank of $\boldsymbol{W}$ must be 2 . The following example shows how to generate the ECBSTBCs for three relay sensors. Consider the ECBSTBC pair with transmission matrix

$$
C_{1}=\left[\begin{array}{ccc}
s_{1} & s_{2} & a s_{2} \\
-s_{2}^{*} & s_{1}^{*} & a s_{1}^{*}
\end{array}\right] .
$$

where $a=\mathrm{e}^{\mathrm{j} 2 \pi m / q}, q$ is the extension level and $m=0,1, \ldots q-1$. The columns and rows of $C_{1}$ denote symbols transmitted from three relay sensors in two signaling intervals, respectively. $C_{1}$ is obtained from the Alamouti code using (1) where

$$
X=\left[\begin{array}{cc}
s_{1} & s_{2} \\
-s_{2}^{*} & s_{1}^{*}
\end{array}\right] \quad W=\left[\begin{array}{lll}
1 & 0 & 0 \\
0 & 1 & a
\end{array}\right] \text {. }
$$

In this fashion, arbitrary number of the ECBSTBCs can be generated by increasing the extension level. In CBSTBC, the number of codes is limited since $q$ is equal to 2 and $a= \pm 1$. It can be shown that the number of possible ECBSTBCs is $q^{\mathrm{N}-2}\left(2^{\mathrm{N}-1}-1\right)$.

For that reason, the fusion center needs $N+d$ feedback bits $(N \geq 3)$ to select any possible ECBSTBCs where $d=\operatorname{ceil}\left\{(N-2) \log _{2} q\right\}-1$. $N-2$ feedback bits are needed to achieve full diversity as in CBSTBCs [14]. The rest of the $d-1$ feedback bits provide coding gain. In CBSTBCs, the extension level is only two, then, the number of available codes is $2^{\mathrm{N}-2}\left(2^{\mathrm{N}-1}-1\right)[14]$. 


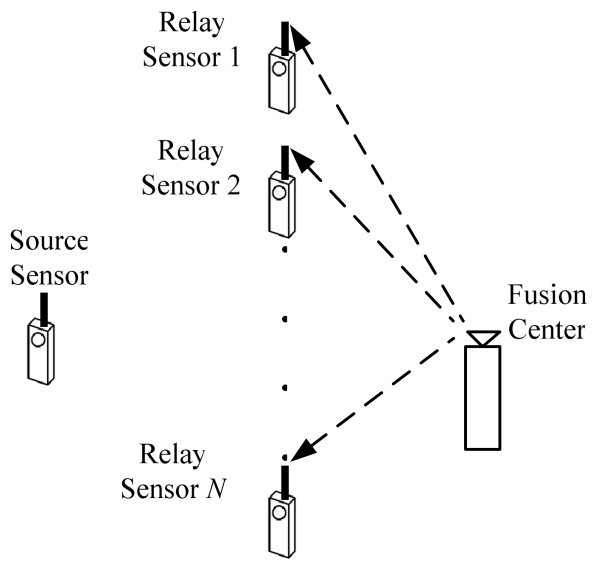

Fig. 1. Frame Initialization phase of the ECBSTBCs
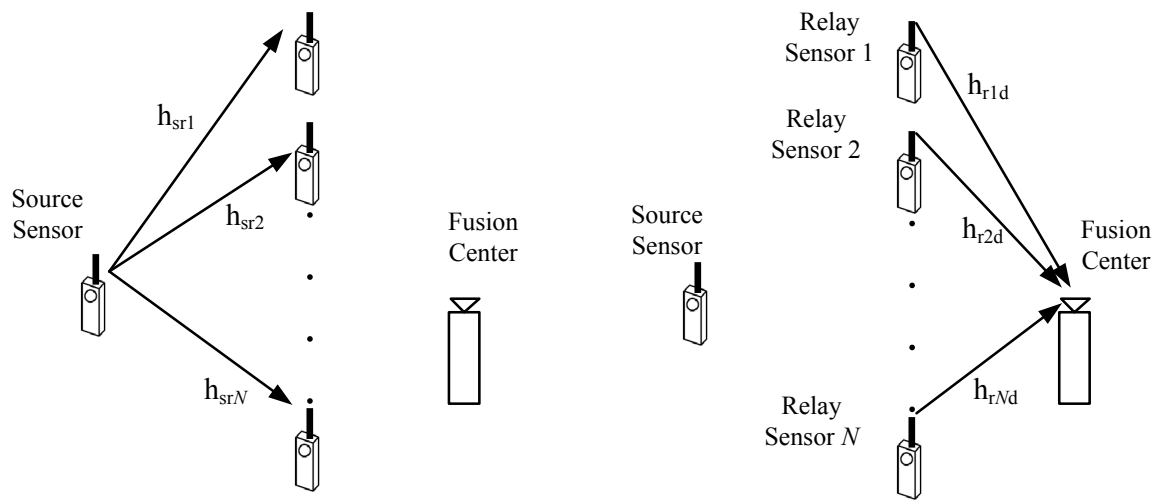

Fig. 2. Broadcast and Cooperation phases of the ECBSTBCs

The ECBSTBCs can be used in wireless sensor networks. The ECBSTBCs contain two phases: Broadcast and cooperation. There are many broadcasts and cooperation phases respectively within a frame. Additionally, each frame includes an initialization phase. In the initialization phase, which occurs at the beginning of the each frame, the fusion center informs the relay sensors about which ECBSTBC would be utilized within the frame as shown in Figure 1. The selected code is fixed over one frame. Figure 2 depicts the broadcast and cooperation phases of the ECBSTBCs. In the broadcast phase, the source sensor transmits symbols $s_{1}$ and $s_{2}$ to the relay sensors and the received signals at the sensor relays can be given as follows

$$
\begin{aligned}
& r_{r i, 1}=\sqrt{P_{1}} h_{s r i} s_{1}+n_{r i, 1} \\
& r_{r i, 2}=\sqrt{P_{1}} h_{s r i} s_{2}+n_{r i, 2} .
\end{aligned}
$$


Here $r_{r i, j}$ is the received signal by the $i$ th relay sensor at the $j$ th symbol interval, $n_{r i, j}$ is the additive white Gaussian noise at the $i$ th relay sensor at the $j$ th symbol interval. Each cooperating relay sensor makes a basic linear transformation to normalize the received signal.

$$
\bar{r}_{r i, 1}=\frac{r_{r i, 1}}{\left|r_{r i, 1}\right|} \quad \bar{r}_{r i, 2}=\frac{r_{r i, 2}}{\left|r_{r i, 2}\right|} .
$$

Here $\bar{r}_{r i, k}$ is the normalized received signal by the $i$ th relay sensor at the $j$ th symbol interval. In the cooperation phase of the ECBSTBCs, the fusion center receives the signal, $\boldsymbol{r}_{\boldsymbol{D}}$,

$$
\boldsymbol{r}_{\boldsymbol{D}}=\sqrt{\frac{P_{2}}{N}} \boldsymbol{C h}_{\boldsymbol{r d}}+\boldsymbol{n}_{\boldsymbol{D}} .
$$

Here $\boldsymbol{h}_{\boldsymbol{r} \boldsymbol{d}}$ is the channel coefficient vector that contains path gains from the relay sensors to the fusion center, $\boldsymbol{n}_{\boldsymbol{D}}$ is additive white Gaussian noise vector whose components are complex zero-mean with variance $\sigma^{2}$, and $\boldsymbol{C}$ is the ECBSTBC matrix.

In [16], distributed space-time coding scenario is analyzed. The optimum power allocation between the source and the relays is chosen to minimize the PEP [16]. For large number of relays which utilize the amplify-and-forward protocol, the optimum power allocation is when the source consumes half of the power and the relays consume the other half. Following a similar analysis carried out in [16] an upper bound for the ECBSTBCs PEP expression is given as,

$$
P\left(s_{k} \rightarrow \hat{s}_{k}\right) \leq E\left(\operatorname{det}^{-1}\left[I_{N}+\frac{P T}{8\left(N+\sum_{i=1}^{N}\left|h_{r i d}\right|^{2}\right)}\left|s_{k}-\tilde{s}_{k}\right|^{2} H_{r d}\right]\right) .
$$

where $P_{T}$ is the total power used in the network and $H_{r d}=\operatorname{diag}\left\{\left|h_{r l d}\right|^{2},\left|h_{r 2 d}\right|^{2}, \ldots,\left|h_{r N d}\right|^{2}\right\}$. Then, the achievable diversity of the ECBSTBCs is $\min \{T, N\}(1-\log \log P / \log P)$ [16]. With the assumption that $T$ is greater or equal to $N$ and $P$ is very large, the diversity gain about $N$ is obtained [16].

\subsection{Three Relay Sensors}

Due to energy efficiency, when three relay sensors are active in the wireless environment, then, $C_{1}, C_{2}$ and $C_{3}$ are available ECBSTBC matrices. These matrices are

$$
C_{1}=\left[\begin{array}{ccc}
s_{1} & s_{2} & a s_{2} \\
-s_{2}^{*} & s_{1}^{*} & a s_{1}^{*}
\end{array}\right] C_{2}=\left[\begin{array}{ccc}
s_{1} & s_{2} & a s_{1} \\
-s_{2}^{*} & s_{1}^{*} & -a s_{2}^{*}
\end{array}\right] \quad C_{3}=\left[\begin{array}{ccc}
s_{1} & a s_{1} & s_{2} \\
-s_{2}^{*} & -a s_{2}^{*} & s_{1}^{*}
\end{array}\right] .
$$

Here $a$ is the coefficient as defined previously. The fusion center selects the ECBSTBC $C_{\mathrm{j}}, \mathrm{j}=1,2,3$ and the feedback bit $a$ that gives the maximum coding gain. Two bits of feedback is needed to select the ECBSTBC matrices and $k$ bit of is needed to select the feedback bit $a$ where $k=\operatorname{ceil}\left\{\log _{2} q\right\}$. 
The decoding of the ECBSTBCs is similar to CBSTBCs [14]. Assume that the $\mathrm{C}_{1}$ matrix gives maximum coding gain. The received signals at fusion center are given as

$$
\begin{aligned}
& r_{D, 1}=\sqrt{\frac{P_{1}}{3}}\left[h_{r 1 d} \bar{r}_{r 1,1}+h_{r 2 d} \bar{r}_{r 2,2}+a h_{r 3 d} \bar{r}_{r 3,2}\right]+\eta_{1} \\
& r_{D, 2}=\sqrt{\frac{P_{1}}{3}}\left[-h_{r 1 d} \bar{r}_{r 1,2}^{*}+h_{r 2 d} \bar{r}_{r 2,1}^{*}+a h_{r 3 d} \bar{r}_{r 3,1}^{*}\right]+\eta_{2} .
\end{aligned}
$$

Here $\eta_{1}$ and $\eta_{2}$ are noise at the fusion center. The fusion center estimates $s_{1}$ and $s_{2}$ by linear processing

$$
\begin{aligned}
& \hat{s}_{1}=h_{r 1 d}^{*} r_{D, 1}+\left(h_{r 2 d}+a h_{r 3 d}\right) r_{D, 2}^{*} \\
& \hat{s}_{2}=\left(h_{r 2 d}^{*}+a h_{r 3 d}^{*}\right) r_{D, 1}^{*}-h_{r 1 d} r_{D, 2}^{*} .
\end{aligned}
$$

Substituting $r_{D, 1}$ and $r_{D, 2}$ in Eq.10,

$$
\begin{aligned}
& \hat{s}_{1}=\sqrt{\frac{P_{2}}{3}}\left[\left(\left|h_{r 1 d}\right|^{2}+\left|h_{r 2 d}\right|^{2}+\left|h_{r 3 d}\right|^{2}+2 \max \left(\operatorname{Re}\left\{a h_{r 2 d}^{*} h_{r 3 d}\right\}, \operatorname{Re}\left\{a h_{r 1 d}^{*} h_{r 3 d}\right\}, \operatorname{Re}\left\{a h_{r 1 d}^{*} h_{r 2 d}\right\}\right)\right)\right] s_{1}+n_{1} . \\
& \hat{s}_{2}=\sqrt{\frac{P_{2}}{3}}\left[\left(\left|h_{r l d}\right|^{2}+\left|h_{r 2 d}\right|^{2}+\left|h_{r 3 d}\right|^{2}+2 \max \left(\operatorname{Re}\left\{a h_{r 2 d}^{*} h_{r 3 d}\right\}, \operatorname{Re}\left\{a h_{r 1 d}^{*} h_{r 3 d}\right\}, \operatorname{Re}\left\{a h_{r l d}^{*} h_{r 2 d}\right\}\right)\right)\right] s_{2}+n_{2}
\end{aligned}
$$

where $n_{1}$ and $n_{2}$ are the noise terms which include the observation noise of the both at the relay sensors and at the fusion center. The contribution of the $2 \max \left(\operatorname{Re}\left\{a h_{r 2 d}^{*} h_{r 3 d}\right\}, \operatorname{Re}\left\{a h_{r 1 d}^{*} h_{r 3 d}\right\}, \operatorname{Re}\left\{a h_{r 1 d}^{*} h_{r 2 d}\right\}\right)$ in Eq.11 will always be positive and the gain will be greater than the sum of the magnitude squares of all path gains $\left(\left|h_{r 1 d}\right|^{2}+\left|h_{r 2 d}\right|^{2}+\left|h_{r 3 d}\right|^{2}\right)$.

\subsection{Four Relay Sensors}

It is assumed that four relay sensors are active in wireless environment, then, 7 different ECBSTBC matrices can be obtained. These matrices are

$$
\begin{gathered}
C_{1}=\left[\begin{array}{cccc}
s_{1} & a s_{1} & b s_{1} & s_{2} \\
-s_{2}^{*} & -a s_{2}^{*} & -b s_{2}^{*} & s_{1}^{*}
\end{array}\right] C_{2}=\left[\begin{array}{cccc}
s_{1} & a s_{1} & s_{2} & b s_{1} \\
-s_{2}^{*} & -a s_{2}^{*} & s_{1}^{*} & -b s_{2}^{*}
\end{array}\right] \\
C_{3}=\left[\begin{array}{cccc}
s_{1} & s_{2} & a s_{1} & b s_{1} \\
-s_{2}^{*} & s_{1}^{*} & -a s_{2}^{*} & -b s_{2}^{*}
\end{array}\right] C_{4}=\left[\begin{array}{cccc}
s_{1} & s_{2} & a s_{2} & b s_{2} \\
-s_{2}^{*} & s_{1}^{*} & a s_{1}^{*} & b s_{1}^{*}
\end{array}\right] \\
C_{5}=\left[\begin{array}{cccc}
s_{1} & a s_{1} & s_{2} & b s_{2} \\
-s_{2}^{*} & -a s_{2}^{*} & s_{1}^{*} & b s_{1}^{*}
\end{array}\right] C_{6}=\left[\begin{array}{cccc}
s_{1} & s_{2} & a s_{1} & b s_{2} \\
-s_{2}^{*} & s_{1}^{*} & -a s_{2}^{*} & b s_{1}^{*}
\end{array}\right] \\
C_{7}=\left[\begin{array}{cccc}
s_{1} & s_{2} & a s_{2} & b s_{1} \\
-s_{2}^{*} & s_{1}^{*} & a s_{1}^{*} & -b s_{2}^{*}
\end{array}\right] .
\end{gathered}
$$

where $a=\mathrm{e}^{\mathrm{j} 2 \pi m / q}$ and $b=\mathrm{e}^{\mathrm{j} 2 \pi m / q}$. The fusion center selects the ECBSTBC matrix $C_{j}$, $j=1, ., 7$ and the coefficients $a$ and $b$ that yields the maximum coding gain. Three bits of feedback is needed to select the ECBSTBC matrix and $(N-2) k$ bits of feedback is needed to select feedback $a$ and $b$ where $k=\operatorname{ceil}\left\{\log _{2} q\right\}$. 
The decoding of the ECBSTBCs is similar to CBSTBCs [14]. After combining and linear processing of received signals, the estimates of the PSK symbols $s_{1}$ and $s_{2}$ can be expressed as follows

$$
\begin{aligned}
& \hat{s}_{1}=\frac{\sqrt{P_{2}}}{2}\left(\begin{array}{ll}
\left|h_{r 1 d}\right|^{2}+\left|h_{r 2 d}\right|^{2}+\left|h_{r 3 d}\right|^{2}+\left|h_{r 4 d}\right|^{2} \\
{\left[\begin{array}{l}
{\left[\operatorname{Re}\left\{a h_{r 1 d}^{*} h_{r 2 d}\right\}+\operatorname{Re}\left\{b h_{r 1 d}^{*} h_{r 3 d}\right\}+\operatorname{Re}\left\{a b^{*} h_{r 2 d} h_{r 3 d}^{*}\right\}\right],} \\
{\left[\operatorname{Re}\left\{a h_{r 1 d}^{*} h_{r 2 d}\right\}+\operatorname{Re}\left\{b h_{r 1 d}^{*} h_{r 4 d}\right\}+\operatorname{Re}\left\{a b^{*} h_{r 2 d} h_{r 4 d}^{*}\right\}\right],} \\
{\left[\operatorname{Re}\left\{a h_{r 1 d}^{*} h_{r 3 d}\right\}+\operatorname{Re}\left\{b h_{r 1 d}^{*} h_{r 4 d}\right\}+\operatorname{Re}\left\{a b^{*} h_{r 3 d} h_{r 4 d}^{*}\right\}\right],} \\
{\left[\operatorname{Re}\left\{a h_{r 2 d}^{*} h_{r 3 d}\right\}+\operatorname{Re}\left\{b h_{r 2 d}^{*} h_{r 4 d}\right\}+\operatorname{Re}\left\{a b^{*} h_{r 3 d} h_{r 4 d}^{*}\right\}\right]} \\
{\left[\operatorname{Re}\left\{a h_{r 1 d}^{*} h_{r 2 d}\right\}+\operatorname{Re}\left\{b h_{r 3 d}^{*} h_{r 4 d}\right\}\right],} \\
{\left[\operatorname{Re}\left\{a h_{r 1 d}^{*} h_{r 3 d}\right\}+\operatorname{Re}\left\{b h_{r 2 d}^{*} h_{r 4 d}\right\}\right],} \\
{\left[\operatorname{Re}\left\{a h_{r 2 d}^{*} h_{r 3 d}\right\}+\operatorname{Re}\left\{b h_{r 1 d}^{*} h_{r 4 d}\right\}\right]}
\end{array}\right)}
\end{array}\right) s_{1}+\omega_{1} \\
& \hat{s}_{2}=\frac{\sqrt{P_{2}}}{2}\left(\begin{array}{ll}
\left|h_{r 1 d}\right|^{2}+\left|h_{r 2 d}\right|^{2}+\left|h_{r 3 d}\right|^{2}+\left|h_{r 4 d}\right|^{2} \\
{\left[\begin{array}{l}
{\left[\operatorname{Re}\left\{a h_{r 1 d}^{*} h_{r 2 d}\right\}+\operatorname{Re}\left\{b h_{r 1 d}^{*} h_{r 3 d}\right\}+\operatorname{Re}\left\{a b^{*} h_{r 2 d} h_{r 3 d}^{*}\right\}\right]} \\
{\left[\operatorname{Re}\left\{a h_{r 1 d}^{*} h_{r 2 d}\right\}+\operatorname{Re}\left\{b h_{r 1 d}^{*} h_{r 4 d}\right\}+\operatorname{Re}\left\{a b^{*} h_{r 2 d} h_{r 4 d}^{*}\right\}\right],} \\
{\left[\operatorname{Re}\left\{a h_{r 1 d}^{*} h_{r 3 d}\right\}+\operatorname{Re}\left\{b h_{r 1 d}^{*} h_{r 4 d}\right\}+\operatorname{Re}\left\{a b^{*} h_{r 3 d} h_{r 4 d}^{*}\right\}\right],} \\
{\left[\operatorname{Re}\left\{a h_{r 2 d}^{*} h_{r 3 d}\right\}+\operatorname{Re}\left\{b h_{r 2 d}^{*} h_{r 4 d}\right\}+\operatorname{Re}\left\{a b^{*} h_{r 3 d} h_{r 4 d}^{*}\right\}\right]} \\
{\left[\operatorname{Re}\left\{a h_{r 1 d}^{*} h_{r 2 d}\right\}+\operatorname{Re}\left\{b h_{r 3 d}^{*} h_{r 4 d}\right\}\right],} \\
{\left[\operatorname{Re}\left\{a h_{r 1 d}^{*} h_{r 3 d}\right\}+\operatorname{Re}\left\{b h_{r 2 d}^{*} h_{r 4 d}\right\}\right],} \\
{\left[\operatorname{Re}\left\{a h_{r 2 d}^{*} h_{r 3 d}\right\}+\operatorname{Re}\left\{b h_{r 1 d}^{*} h_{r 4 d}\right\}\right]}
\end{array}\right)}
\end{array}\right) s_{2}+\omega_{2}
\end{aligned}
$$

where $\omega_{1}$ and $\omega_{2}$ are the noise terms which include the observation noise of both at the relay sensors and at the fusion center. The contribution of the coding gain in Eq.13-14 will always be positive and the gain will be greater than the sum of the magnitude squares of all path gains $\left(\left|h_{r 1 d}\right|^{2}+\left|h_{r 2 d}\right|^{2}+\left|h_{r 3 d}\right|^{2}+\left|h_{r 4 d}\right|^{2}\right)$.

\section{Performance Evaluations}

In the cooperative communication transmitting only from selected relays is called distributed transmit antenna selection (DTAS) [17] which is an alternative approach to the ECBSTBCs. The criterion in selecting a single relay sensor is the best instantaneous relay-fusion center channel gain [18], and this is called as transmit sensor selection (TSS $N: 1$ ). To maximize SNR at the fusion center, two relay sensors are chosen out of all the available transmit relay sensors and then the relay sensors transmit the received signals using the Alamouti scheme [19]. In the simulations, the best relay 
pair which has best instantaneous relay-fusion center channel pair is selected. This is called as transmit sensor selection with Alamouti (TSS N:2).

The bit error probabilities of the proposed ECBSTBC sets are evaluated for quaternary phase-shift keying (QPSK) modulation by computer simulations. A frame of 130 symbols is used. For meaningful comparison, the total transmission power and bandwidth are fixed. Namely, in the broadcast phase; the source sensor transmits with full power but in the cooperation phase, the power is divided equally among relay sensors.

For comparison purposes, the bit error rate (BER) curves of the transmit sensor selection with Alamouti's scheme and transmit sensor selection are also included in Figs. 3-6.

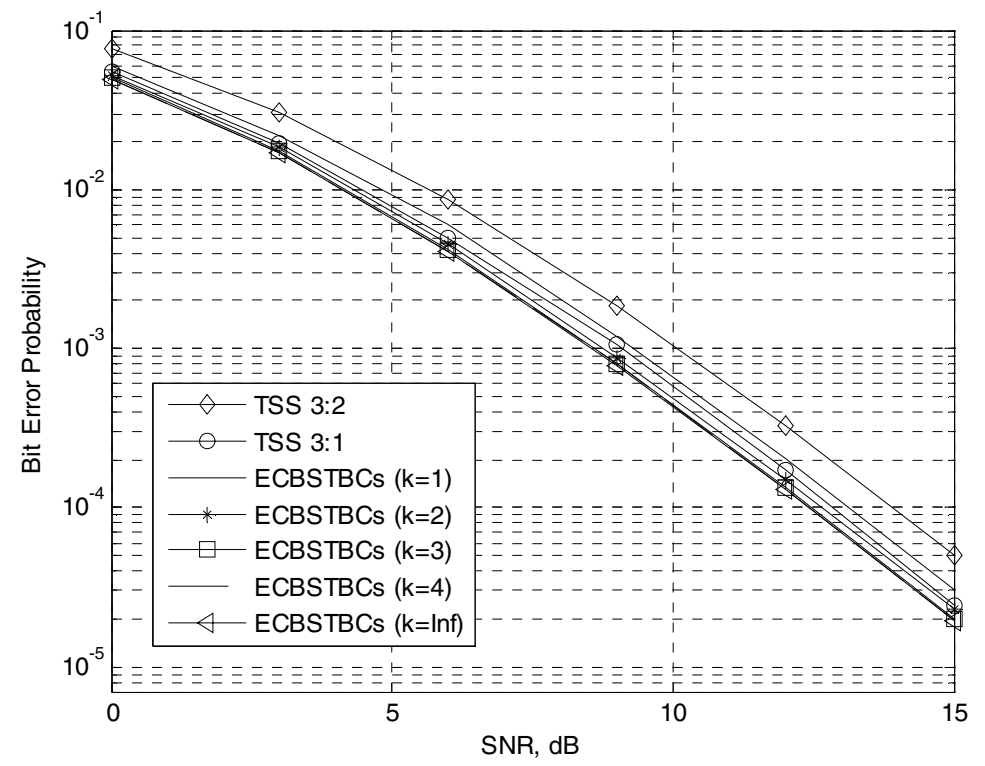

Fig. 3. Bit-error probability performance of sensor networks when three relay sensors are active and the source sensor-relay sensors' channels are ideal

Figure 3 presents the bit error probabilities of the ECBSTBC with one to four bits extension of feedback and ideal (infinite) feedback for three relay sensors. We assume that the source-relay sensor channels are ideal to be able to investigate pure coding performance. Compared to the transmit sensor selection with Alamouti's scheme (TSS 3:2), the ECBSTBC with one bit extension (ECBSTBCs $(\mathrm{k}=1)$ ) provides an SNR advantage of approximately $0.74 \mathrm{~dB}$ for a BER value of $P_{\mathrm{b}}=10^{-4}$. However, transmit sensor selection (TSS 3:1) provides approximately $0.31 \mathrm{~dB}$ better performance than ECBSTBC with one bit extension due to limited coding gain. If we extend feedback by two bits (ECBSTBCs $(\mathrm{k}=2)$ ), the ECBSTBCs provide approximately $0.21 \mathrm{~dB}$ better performance than the TSS. Thus, on the basis of the same SNR, the ECBSTBCs outperform the transmit sensor selection. If three bits of extension 
(ECBSTBCs $(\mathrm{k}=3)$ ) is available for feedback, an additional $0.13 \mathrm{~dB}$ coding gain is available. Performance of four bits extension of feedback (ECBSTBCs $(\mathrm{k}=4)$ ) is similar to ideal feedback (ECBSTBCs ( $\mathrm{k}=\mathrm{Inf}$.)). As expected, when the number of extension level is increased, the bit error performance of ECBSTBC is improved.

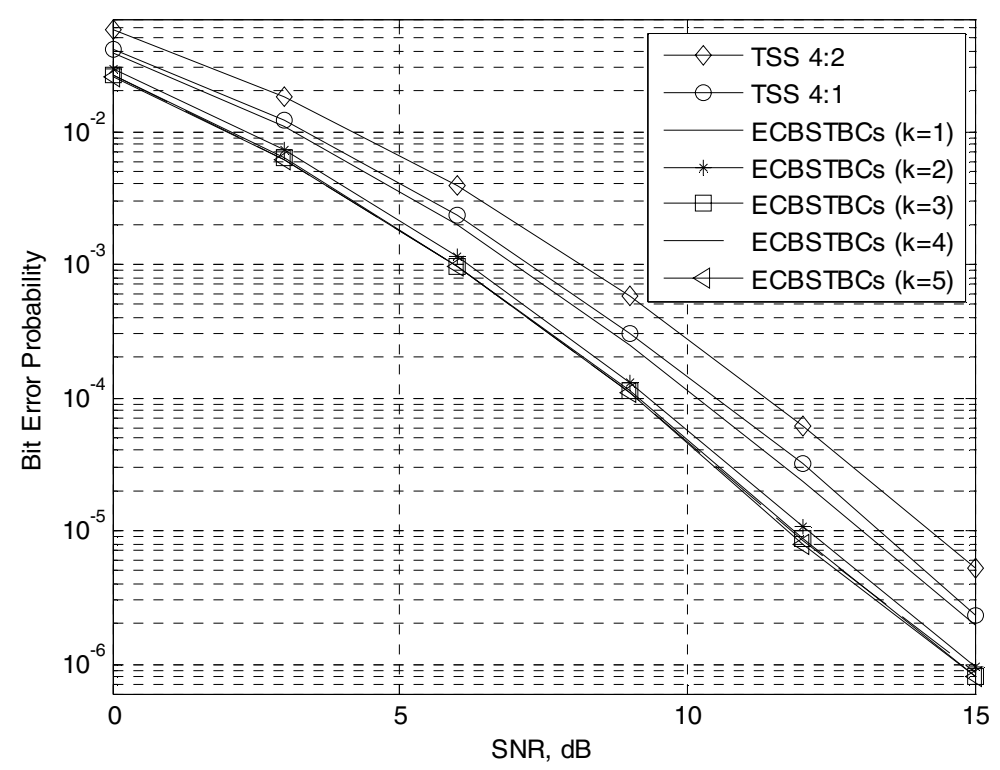

Fig. 4. Bit-error probability performance of sensor networks when four relay sensors are active and the source sensor-relay sensors' channels are ideal

Figure 4 presents the bit error probabilities of the ECBSTBC with one to five bits extension of feedback bits for four relay sensors. Compared to the TSS with Alamouti's scheme (TSS 4:2), the TSS (TSS 4:1) provides an SNR advantage of approximately $0.89 \mathrm{~dB}$ for a BER value of $\mathrm{Pb}=10^{-5}$. However, the ECBSTBCs with one bit extension (ECBSTBCs $(\mathrm{k}=1)$ ) provides approximately $0.29 \mathrm{~dB}$ better performance than the TSS. If we extend the feedback information by two bits (ECBSTBCs $(\mathrm{k}=2)$ ), the ECBSTBC provides approximately $0.94 \mathrm{~dB}$ better performance than the ECBSTBCs with one bit extension. If three bits extension of feedback is available, an additional $0.23 \mathrm{~dB}$ coding gain is obtained. Four bits and five bits extension of feedback yields approximately similar performance. As expected, as the number of available codes is increased the coding gain is increased.

In the sequel, we assumed that the source-relay channels are not ideal, nevertheless, are better quality than relay-fusion center channels whose difference is quantified by differential signal-to-noise ratio (DSNR). In the Figure 5-6, DSNR is assumed to be $15 \mathrm{~dB}$ for three and four relay sensors. Once again, the ECBSTBCs provide better performance than the sensor selection schemes and the CBSTBCs. 


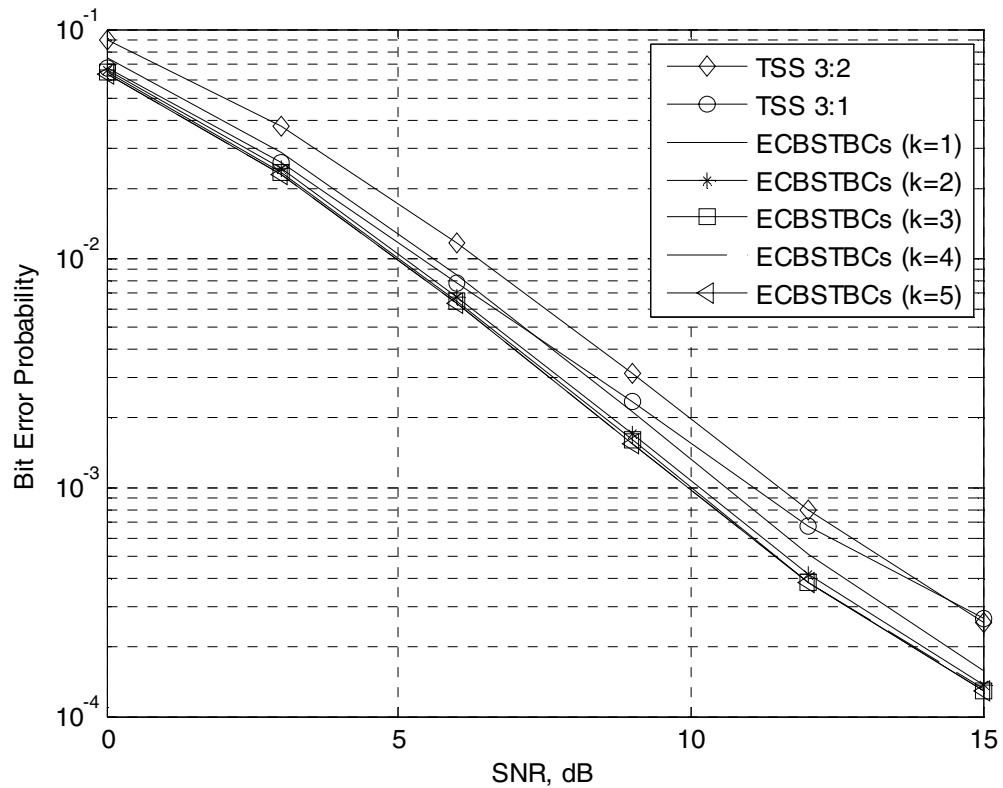

Fig. 5. Bit-error probability performance of sensor networks when three relay sensors are active and the source sensor-relay sensors' channels are 15dB DSNR

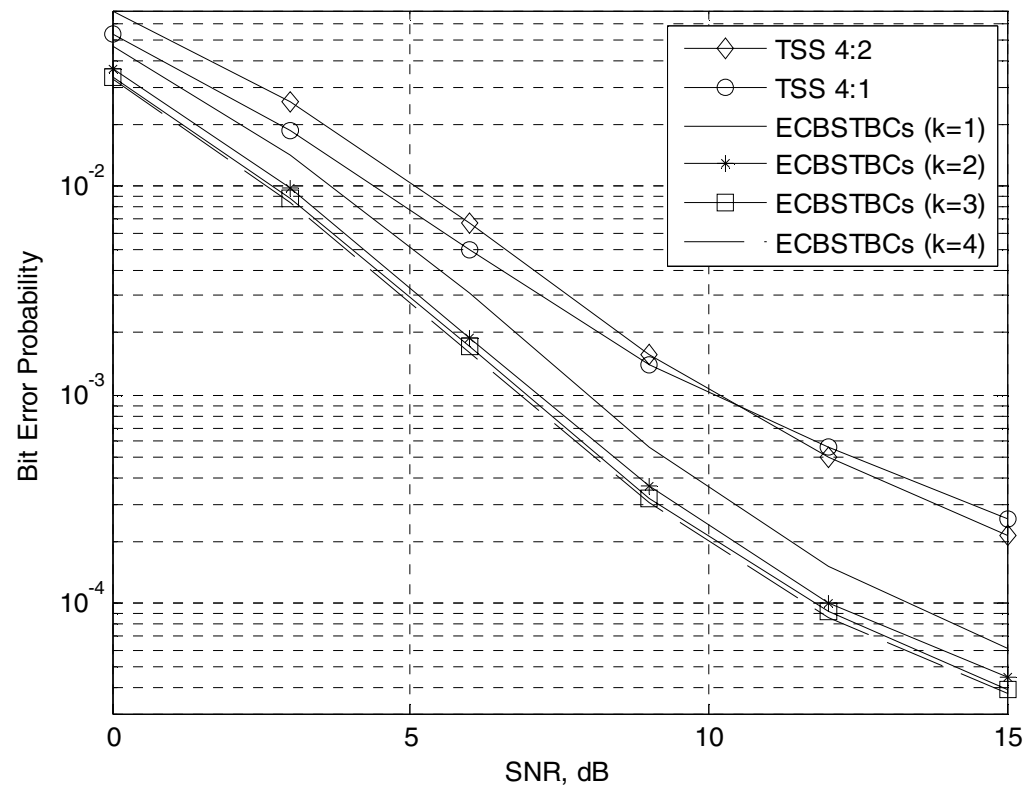

Fig. 6. Bit-error probability performance of sensor networks when four relay sensors are active and the source sensor-relay sensors' channels are 15dB DSNR 


\section{Conclusions}

Network lifetime extension is better achieved via uniform energy distribution over a wireless sensor network. One of the promising methods for effective communication in wireless sensor networks is the CBSTBC which provides full diversity and distributes the energy consumption over a larger part of the network as opposed to sensor selection methods. In this work, we improve the performance of the CBSTBCs by means of extending the number of available codes to provide improved coding gain. Simulations, demonstrated that four bit extension of feedback gives approximately ideal feedback performance. In addition, the ECBSTBCs have better signal-to-noise ratio improvement compared to sensor selection schemes. Consequently, we conclude that the ECBSTBC is a useful tool to increase the QoS of wireless sensor networks.

\section{References}

1. Vucetic, B., Yuan, J.: Space-Time Coding. John Wiley \& Sons, Chichester (2003)

2. Liu, L., Lim, M.-S.: Selective receiver switching scheme for space time block coding with full code rate and non-orthogonal design. IET Commun. 2(5), 664-672 (2008)

3. Yousafzai, A.K., Nakhai, M.R.: Reduced complexity detection technique for layered space time block coded multiple-input multiple-output orthogonal frequency division multiplexing. IET Commun. 3(1), 115-122 (2009)

4. Çelebi, M.E., Şahin, S., Aygölü, Ü.: Balanced space-time block coding: An efficient way to increase diversity with feedback. IEE Proc-Commun. MIMO Wireless and Mobile Commun. 153(4) (2006)

5. Tarokh, V., Jafarkhani, H., Calderbank, A.R.: Space-time block codes from orthogonal designs. IEEE Transactions on Information Theory 45, 1456-1467 (1999)

6. Jafarkhani, H.: A quasi-orthogonal space-time block code. IEEE Trans. Commun. 49, 1-4 (2001)

7. Tirkkonen, O., Hottinen, A.: Complex space-time block codes for four Tx antennas. In: Proc. GLOBECOM, pp. 1005-1009. IEEE Press, San Fransisco (2000)

8. Su, W., Xia, X.G.: On space-time block codes from complex orthogonal designs. Wirel. Pers. Commun. 25, 1-26 (2003)

9. Laneman, J.N., Wornell, G.W., Tse, D.N.C.: An efficient protocol for realizing cooperative diversity in wireless networks. In: Proc. IEEE ISIT, Washington D.C. IEEE Press, Los Alamitos (2001)

10. Sendonaris, A., Erkip, E., Aazhang, B.: User cooperation diversity part I: System description. IEEE Trans. Commun. 51(11), 1927-1938 (2003)

11. Sendonaris, A., Erkip, E., Aazhang, B.: User cooperation diversity part II: Implementation aspects and performance analysis. IEEE Trans. Commun. 51(11), 1939-1948 (2003)

12. Nabar, R.U., Bolcskei, H., Kneubuhler, F.W.: Fading relay channels: Performance limits and space-time signal design. IEEE J. Select. Areas Commun., 1099-1109 (2004)

13. Tang, Y., Valenti, M.C.: Coded transmit macrodiversity: Block space-time codes over distributed antennas. In: IEEE Veh. Technol. Conference, pp. 1435-1438. IEEE Press, Los Alamitos (2001)

14. Ekşim, A., Çelebi, M.E.: Diversity enhancement with cooperative balanced space-time block coding. In: Proc. of the 18th Annual IEEE International Symposium on Personal, Indoor and Mobile Radio Communications (PIMRC 2007). IEEE Press, Athens (2007) 
15. Alamouti, S.M.: A simple transmit diversity technique for wireless communications. IEEE J. Select. Areas Commun. 16, 1451-1458 (1998)

16. Jing, Y., Hassibi, B.: Distributed space-time coding in wireless relay networks. IEEE Trans. on Wirel. Commun. 5(12) (2006)

17. Michalopoulos, D.S., Karagiannidis, G.K., Tsiftsis, T.A., Mallik, R.K.: Distributed transmit antenna selection (DTAS) under performance or energy consumption constraints. IEEE Trans. Wireless Commun. 7, 1168-1173 (2008)

18. Luo, J., Blum, R.S., Greenstein, L.J., Haimovich, A.M.: Link-failure probabilities for practical cooperative relay networks. In: Proc. of the IEEE vehicular Technology Conference (VTC 2005 Spring). IEEE Press, Stockholm (2005)

19. Gore, D., Paulraj, A.: MIMO antenna subset selection with space-time coding. IEEE Trans. Signal Process 50, 2580-2588 (2002) 